\title{
NON-DEGENERACY OF PERTURBED SOLUTIONS OF SEMILINEAR PARTIAL DIFFERENTIAL EQUATIONS
}

\author{
Robert Magnus and Olivier Moschetta \\ University of Iceland, The University Science Institute \\ Dunhaga 3, 107-Reykjavik, Iceland; robmag@hi.is \\ University of Iceland, The University Science Institute \\ Dunhaga 3, 107-Reykjavik, Iceland; oms3@hi.is
}

\begin{abstract}
The equation $-\Delta u+F(V(\varepsilon x), u)=0$ is considered in $\mathbf{R}^{n}$. For small $\varepsilon>0$ it is shown to possess, under appropriate conditions, a non-degenerate solution $u_{\varepsilon}$ in $H^{2}\left(\mathbf{R}^{n}\right)$. It is shown that the linearised operator $T_{\varepsilon}$ at the solution satisfies $\left\|T_{\varepsilon}^{-1}\right\|=O\left(\varepsilon^{-2}\right)$ as $\varepsilon \rightarrow 0$.
\end{abstract}

\section{Introduction}

In this paper we consider the question of non-degeneracy of certain solutions of a partial differential equation. A solution is non-degenerate when the linearised problem at the solution, in appropriate function spaces, defines an invertible linear operator. Throughout this article, by an invertible operator, with specified Banach spaces as domain and codomain, we shall mean a linear surjective homeomorphism.

The notion of non-degeneracy depends on the choice of spaces. In this paper we shall be concerned with problems that can be posed using the Sobolev spaces $W^{2,2}\left(\mathbf{R}^{n}\right)$ and $L^{2}\left(\mathbf{R}^{n}\right)$ as domain and codomain respectively. For conciseness we denote these spaces by $H^{2}$ and $L^{2}$ respectively. The same problems might be posable in spaces of classically differentiable functions and the question of non-degeneracy in this setting can also arise. There are connections between the two notions of nondegeneracy which do not seem to have been fully explored. In this paper we shall study non-degeneracy for Sobolev spaces only.

It seems safe to assume that the applications of non-degeneracy, once established, are many. We could mention the stable behaviour of non-degenerate solutions under perturbations guaranteed by the implicit function theorem. Moreover for equations of the type we shall consider, non-degenerate solutions give rise to multibump solutions, see for example [1] (using spaces of differentiable functions), or [3] (using Sobolev spaces).

Let us first consider the problem

$$
-\Delta u+F(u)=0
$$

in $\mathbf{R}^{n}$. Under conditions on $F$ to be specified the non-linear operator $\Gamma(u)=-\Delta u+$ $F(u)$ is well-defined from $H^{2}$ to $L^{2}$ and will have a well-defined Fréchet derivative, the linear operator $v \mapsto D \Gamma(u) v=-\Delta v+\frac{d F}{d u}(u) v$.

Let us assume that we have a solution $\phi$ of (1.1). It is highly implausible for $\phi$ to be a non-degenerate solution as the partial derivatives $D_{k} \phi$ will be in the kernel of

doi:10.5186/aasfm.2010.3505

2000 Mathematics Subject Classification: 35J60.

Key words: Non-degeneracy, perturbed solution, semilinear PDE.

The second author was supported by a grant from the University of Iceland Research Fund. 
$D \Gamma(\phi)$ if they lie in $H^{2}$. Similarly, since the problem (1.1) commutes with rotations, we expect the functions $\nabla \phi(x) \cdot T x$ to be in the kernel whenever $T$ is a skew-symmetric matrix. However these functions will be 0 if $\phi$ is spherically symmetric.

We shall say that a spherically symmetric solution $\phi$ is quasi-non-degenerate if the partial derivatives $D_{j} \phi(x)$ belong to $H^{2}$, they are linearly independent, span the kernel of $D \Gamma(\phi)$, and the range of $D \Gamma(\phi)$ is the orthogonal complement in $L^{2}$ to its kernel.

Quasi-non-degenerate solutions are easy to construct in one dimension. We consider $-u^{\prime \prime}+F(u)=0$ where $F$ is a smooth function such that $F(0)=0, F^{\prime}(0)>0$ and $\Phi(u)=-\int F(u) d u$ satisfies $\sup _{u>0} \Phi(u)>\Phi(0)$. Then the solution $\phi(x)$ is quasi-non-degenerate where $x \mapsto\left(\phi(x), \phi^{\prime}(x)\right)$ is the phase-plane trajectory in the region $u>0$ which tends to the saddle point $(0,0)$ as $x \rightarrow \pm \infty$.

In higher dimensions a range of quasi-non-degenerate solutions is known for the equation

$$
-\Delta u+u-u^{p}=0
$$

More precisely it is known that the ground state solution, defined to be the solution with minimum energy $\int\left(\frac{1}{2}|\nabla u|^{2}+\frac{1}{2} u^{2}-\frac{1}{p+1} u^{p+1}\right) d x$, exists and is quasi-nondegenerate for all integers $p>1$ if $n=1,2$ and for $1<p<(n+2) /(n-2)$ if $n>2$. See the papers [2], [5] and [7].

The possibility arises of obtaining non-degenerate solutions by perturbing (1.1), when a quasi-non-degenerate solution is known, to a problem explicitly containing $x$. Various perturbation schemes have been studied, for example that of [3, Sections 4, 5], which generates non-degenerate solutions to a more general equation of the type $-\Delta u+F(x, u, \nabla u)=0$. Another paper [4] considered perturbations of a different nature. Suppose we have a one-parameter continuum of problems

$$
-\Delta u+F(a, u)=0
$$

where $a$ belongs to the real interval $I$, and suppose for each value $a \in I$ we have a quasi-non-degenerate solution $\phi_{a}(x)$. An example showing how such a continuum can be constructed is given in Section 4. Now we perturb (1.3) to

$$
-\Delta u+F(V(\varepsilon x), u)=0
$$

where $V(x)$ is a function with range in $I$ and $\varepsilon>0$. The difficulty of this scheme arises from the weak nature of the convergence to a problem of the form (1.3) as $\varepsilon \rightarrow 0$.

In the previous paper [4, Section 3] it was shown how to obtain a solution $u_{\varepsilon}$ in $H^{2}$ of (1.4) for all sufficiently small $\varepsilon$, say, for $0<\varepsilon<\varepsilon_{0}$, and its asymptotic form was described. This is a so-called single-bump solution and it is asymptotic to $\phi_{a}\left(x-\frac{b}{\varepsilon}\right)$ (for a certain $b$ and $a=V(b)$ ) as $\varepsilon \rightarrow 0$. These results are summarised in Section 2, Theorem 2.6. The question of non-degeneracy of the solution $u_{\varepsilon}$ was not studied in [4]. The main object of this paper is provide a clear proof of non-degeneracy for the single-bump solutions of (1.4) together with an estimate of the blow-up of the inverse of the derivative as $\varepsilon \rightarrow 0$. In fact we shall show that the linearised operator

$$
T_{\varepsilon}:=-\Delta+\frac{\partial F}{\partial u}\left(V(\varepsilon x), u_{\varepsilon}\right): H^{2} \rightarrow L^{2}
$$


is invertible for sufficiently small $\varepsilon>0$. Moreover, its inverse satisfies an estimate

$$
\left\|T_{\varepsilon}^{-1}\right\|=O\left(\frac{1}{\varepsilon^{2}}\right)
$$

as $\varepsilon$ tends to 0 .

The precise version of this result, Theorem 3.1, is stated and proved in Section 3. The proof consists of two distinct and independent steps:

1. It is shown that $T_{\varepsilon}$ is a Fredholm operator of index 0 .

2. It is shown that for every sequence $\varepsilon_{\nu}$ with limit 0 and for every bounded sequence $v_{\nu} \in H^{2}$ such that $\varepsilon_{\nu}^{-2} T_{\varepsilon_{\nu}} v_{\nu} \rightarrow 0$ in $L^{2}$, a subsequence of $v_{\nu}$ tends to 0 in $H^{2}$.

The first step shows that $T_{\varepsilon}$, if injective, is also surjective. The second, and by far lengthier step, shows that $T_{\varepsilon}$ is injective and its inverse, considered as an operator defined on its range, satisfies the claimed estimate.

The proof of the second step considers a decomposition $v_{\nu}\left(\cdot-t_{\nu}\right)=\sigma_{\nu} \cdot \nabla \phi_{a}+w_{\nu}$ where $\sigma_{\nu} \in \mathbf{R}^{n}, \phi_{a}$ is the ground state solution occurring in the asymptotic form of $u_{\varepsilon}$, the function $w_{\nu}$ is orthogonal to the partial derivatives of $\phi_{a}$, and $t_{\nu}$ a certain translation vector. It is then shown that a subsequence can be found for which $\sigma_{\nu} \rightarrow 0$ and $w_{\nu} \rightarrow 0$ in $H^{2}$. Hilbert space methods, for example weak compactness of the unit ball, and self-adjointness of the operator $T_{\varepsilon}$ and related operators, weigh heavily in the proof.

\section{Principal assumptions}

In this section we state clearly the conditions to be imposed on $F, V$ and $\phi_{a}$. We summarise material from [4] that will be needed. We also define precisely the solution $u_{\varepsilon}$ referred to in the introduction.

Properties of $F$. We assume that $F$ is a $C^{2}$ map satisfying the following growth conditions:

$$
\begin{aligned}
|F(a, u)|,\left|\frac{\partial F}{\partial a}(a, u)\right|,\left|\frac{\partial^{2} F}{\partial a^{2}}(a, u)\right| & \leq C\left(|u|+|u|^{\alpha_{1}}\right), \\
\left|\frac{\partial F}{\partial u}(a, u)\right|,\left|\frac{\partial^{2} F}{\partial u \partial a}(a, u)\right| & \leq C\left(1+|u|^{\alpha_{2}}\right), \\
\left|\frac{\partial^{2} F}{\partial u^{2}}(a, u)\right| & \leq C\left(1+|u|^{\alpha_{3}}\right),
\end{aligned}
$$

where $C$ is chosen uniformly for $a$ in a bounded interval and the exponents $\alpha_{i}$ are non-negative (in addition $\alpha_{1} \geq 1$ ). No upper limits are placed on $\alpha_{i}$ if $n \leq 4$ whereas for $n \geq 5$ we assume that

$$
\alpha_{1} \leq \frac{n}{n-4}, \quad \alpha_{2} \leq \frac{4}{n-4}, \quad \alpha_{3}<\frac{8-n}{n-4}
$$


Under these growth conditions $F, \frac{\partial F}{\partial a}, \frac{\partial^{2} F}{\partial a^{2}}, \frac{\partial F}{\partial u}, \frac{\partial^{2} F}{\partial u \partial a}$ and $\frac{\partial^{2} F}{\partial u^{2}}$ define Nemitskii operators

$$
\begin{aligned}
\boldsymbol{F}, \boldsymbol{F}_{a}, & \boldsymbol{F}_{a a}: L^{\infty} \times H^{2} \rightarrow L^{2}, \\
\boldsymbol{F}_{u}, & \boldsymbol{F}_{u a}: L^{\infty} \times H^{2} \rightarrow \mathscr{L}\left(H^{2}, L^{2}\right), \\
\boldsymbol{F}_{u u}: & L^{\infty} \times H^{2} \rightarrow \mathscr{L}_{2}\left(H^{2} \times H^{2}, L^{2}\right)
\end{aligned}
$$

by means of

$$
\boldsymbol{F}(m, u)=F(m, u), \boldsymbol{F}_{u}(m, u) v=\frac{\partial F}{\partial u}(m, u) v
$$

and so on. In these formulas we use $\mathscr{L}_{k}$ to denote the appropriate space of symmetric $k$-linear mappings.

Under these conditions, the Nemitskii operators induced by $F$ and its derivatives have the following boundedness property (see [4]):

Lemma 2.1. The maps $\boldsymbol{F}, \boldsymbol{F}_{a}, \boldsymbol{F}_{a a}, \boldsymbol{F}_{u}, \boldsymbol{F}_{u a}$ and $\boldsymbol{F}_{u u}$ map bounded subsets of $L^{\infty} \times H^{2}$ to bounded subsets of the appropriate function or operator space.

We shall often consider sequences of functions indexed by $\nu$ (" $n$ " is reserved for the dimension of $\mathbf{R}^{n}$ ). It is understood that $\nu$ is an integer and limits, where they occur, are for $\nu \rightarrow \infty$.

The following convergence properties were proved in [4] and will be used repeatedly later on.

Lemma 2.2. Let $m_{\nu} \in L^{\infty}$ be a bounded sequence that tends pointwise to $m \in L^{\infty}$. Let $u_{\nu}$ in $H^{2}$ converge to $u \in H^{2}$ and let $v, w \in H^{2}$. Then

$$
\begin{aligned}
& \boldsymbol{F}\left(m_{\nu}, u_{\nu}\right) \rightarrow \boldsymbol{F}(m, u), \boldsymbol{F}_{a}\left(m_{\nu}, u_{\nu}\right) \rightarrow \boldsymbol{F}_{a}(m, u), \boldsymbol{F}_{u}\left(m_{\nu}, u_{\nu}\right) v \rightarrow \boldsymbol{F}_{u}(m, u) v, \\
& \boldsymbol{F}_{u u}\left(m_{\nu}, u_{\nu}\right)(v, w) \rightarrow \boldsymbol{F}_{u u}(m, u)(v, w) .
\end{aligned}
$$

Lemma 2.3. Let $m_{\nu} \in L^{\infty}$ be a bounded sequence that tends pointwise to $m \in L^{\infty}$ and let $u_{\nu} \in H^{2}$ converge weakly to $u \in H^{2}$. Then, for any bounded sequence $v_{\nu} \in H^{2}$,

$$
\boldsymbol{F}_{u}\left(m_{\nu}, u_{\nu}\right) v_{\nu}-\boldsymbol{F}_{u}(m, u) v_{\nu} \longrightarrow 0
$$

in the weak topology on the dual of $H^{2}$.

Lemma 2.4. Let $m_{\nu}$ be a bounded family in $L^{\infty}$, and $u_{\nu}, v_{\nu}$ and $w_{\nu}$ be bounded sequences in $H^{2}$ such that either

1. $u_{\nu}-v_{\nu}$ is convergent in $H^{2}$ and $w_{\nu}$ converges weakly to 0 , or

2. $u_{\nu}-v_{\nu}$ converges weakly to 0 in $H^{2}$ and $w_{\nu}$ is convergent. Then

$$
\left(\boldsymbol{F}_{u}\left(m_{\nu}, u_{\nu}\right)-\boldsymbol{F}_{u}\left(m_{\nu}, v_{\nu}\right)\right) w_{\nu} \rightarrow 0
$$

in $L^{2}$. Furthermore, $\boldsymbol{F}_{u}(m, u)-\boldsymbol{F}_{u}(m, v)$ is a compact operator for each $m \in L^{\infty}$, and $u, v \in H^{2}$.

Properties of $\phi_{a}$. The function $\phi_{a}(x)$ is a solution to $-\Delta u+F(a, u)=0$ in $H^{2}$ and has the following properties:

1. $\phi_{a}(x)=\Phi_{a}(r)$ is spherically symmetric.

2. $\int \frac{\partial F}{\partial a}\left(a, \Phi_{a}(r)\right) \Phi_{a}^{\prime}(r) r d x \neq 0$.

3. $\phi_{a}$ and its first derivatives have exponential decay. 
4. $\phi_{a}$ is a quasi-non-degenerate solution, that is, the operator

$$
-\Delta+\frac{\partial F}{\partial u}\left(a, \phi_{a}(x)\right): H^{2} \rightarrow L^{2}
$$

has as its kernel the space spanned by the $n$ partial derivatives $D_{j} \phi_{a}(x)$, which are assumed to be independent, and its range is the space in $L^{2}$ orthogonal to its kernel. This implicitly says that the partial derivatives belong to $H^{2}$.

These properties hold in the model case of the non-linear Schrödinger equation (1.2) described in the introduction, see [2], [5] and [7].

Properties of $V$. The function $V$ is $C^{2}$ with its range in the interval $I$. It and its first partial derivatives are bounded, while its second partial derivatives have polynomial growth.

Positivity assumption. There exists $\delta>0$ such that

$$
\frac{\partial F}{\partial u}(a, 0)>\delta
$$

for all $a$ in the range of $V$.

For later reference we shall need a version of Wang's Lemma (see [6, 3]):

Lemma 2.5. Let $f_{\nu}$ be a family of measurable functions such that

$$
0<\delta<f_{\nu}(x)<K
$$

for all $\nu$ and constants $\delta$ and $K$. Let $\mu_{\nu}$ be a sequence of non-negative numbers and let $v_{\nu}$ be a sequence in $H^{2}$ such that

$$
-\Delta v_{\nu}+\left(f_{\nu}(x)+\mu_{\nu}\right) v_{\nu} \rightarrow 0
$$

in $L^{2}$. Then $v_{\nu} \rightarrow 0$ in $H^{2}$.

Under these conditions the following theorem, proved in [4], holds.

Theorem 2.6. Let $b$ be a non-degenerate critical point of $V$ and let $a=V(b)$. Then, for sufficiently small $\varepsilon>0$, the equation $-\Delta u+F(V(\varepsilon x), u)=0$ has a solution of the form

$$
u_{\varepsilon}(x)=\phi_{a}\left(x-\frac{b}{\varepsilon}+s_{\varepsilon}\right)+\varepsilon^{2} w_{\varepsilon}\left(x-\frac{b}{\varepsilon}+s_{\varepsilon}\right)
$$

where $s_{\varepsilon} \in \mathbf{R}^{n}, w_{\varepsilon} \in H^{2}$ and $w_{\varepsilon}$ is orthogonal in $L^{2}$ to the partial derivatives $D_{j} \phi_{a}$. Both $s_{\varepsilon}$ and $w_{\varepsilon}$ depend continuously on $\varepsilon$. As $\varepsilon$ tends to $0, s_{\varepsilon}$ tends to 0 and $w_{\varepsilon}$ tends to a computable function $\eta \in H^{2}$, which is the unique solution $v=\eta(x)$ orthogonal to the partial derivatives $D_{j} \phi_{a}$ of the problem

$$
-\Delta v+\frac{\partial F}{\partial u}\left(a, \phi_{a}(x)\right) v=-\frac{1}{2} \frac{\partial F}{\partial a}\left(a, \phi_{a}(x)\right)(H(b) x \cdot x)
$$

where $H(b)$ is the Hessian matrix of $V$ at the point $b$. Finally the solution $u_{\varepsilon}$ is the unique one possessing these asymptotic properties.

\section{Non-degeneracy of the solutions}

Now that we have defined $u_{\varepsilon}$ we can state precisely the main conclusion of this paper. 
Theorem 3.1. There exists $\varepsilon_{0}>0$ such that for $0<\varepsilon<\varepsilon_{0}$ the solution $u_{\varepsilon}$ obtained under the conditions of Theorem 2.6 is non-degenerate, that is, the operator

$$
T_{\varepsilon}:=-\Delta+\frac{\partial F}{\partial u}\left(V(\varepsilon x), u_{\varepsilon}\right)
$$

from $H^{2}$ to $L^{2}$ is invertible. Moreover, we have the following bound on its inverse:

$$
\left\|T_{\varepsilon}^{-1}\right\| \leq C\left(\frac{1}{\varepsilon^{2}}\right)
$$

where $C$ is independent of $\varepsilon$.

Proof. We refer the reader to the outline given in Section 1. The proof is in two rather unequal steps. The first step is to show that $T_{\varepsilon}$ is a Fredholm operator of index 0 .

Let

$$
A_{\varepsilon}:=-\Delta+\frac{\partial F}{\partial u}(V(\varepsilon x), 0): H^{2} \rightarrow L^{2}
$$

By the positivity assumption $A_{\varepsilon}$ is a self-adjoint operator with domain $H^{2}$ satisfying $A_{\varepsilon}>-\Delta+\delta$, and hence an invertible operator from $H^{2}$ to $L^{2}$. Now $T_{\varepsilon}$ is a compact perturbation of $A_{\varepsilon}$, since $T_{\varepsilon}-A_{\varepsilon}$ is given by multiplication by

$$
f(x):=\frac{\partial F}{\partial u}\left(V(\varepsilon x), u_{\varepsilon}\right)-\frac{\partial F}{\partial u}(V(\varepsilon x), 0)
$$

and therefore defines a compact operator from $H^{2}$ to $L^{2}$ by Lemma 2.4. Hence $T_{\varepsilon}$ is a Fredholm operator of index 0 . It follows that if $T_{\varepsilon}$ is injective it is also surjective.

We turn to the second step as outlined in Section 1. We wish to show that for every sequence $\varepsilon_{\nu}$ with limit 0 and for every bounded sequence $v_{\nu} \in H^{2}$ such that $\varepsilon_{\nu}^{-2} T_{\varepsilon_{\nu}} v_{\nu} \rightarrow 0$ in $L^{2}$ a subsequence of $v_{\nu}$ tends to 0 in $H^{2}$. That this implies the required estimate for the inverse $T_{\varepsilon}^{-1}$ is a consequence of a simple functional analytic lemma.

Lemma 3.2. Let $\left(\Gamma_{\varepsilon}\right)_{0<\varepsilon<\varepsilon_{0}}$ be a family of bounded linear operators from a Banach space $E$ to a Banach space $F$. Assume it satisfies the following condition:

(C) If $\varepsilon_{\nu}$ is a sequence in the interval $] 0, \varepsilon_{0}\left[\right.$ with limit 0 and $x_{\nu}$ a bounded sequence in $E$ such that $\lim \Gamma_{\varepsilon_{\nu}} x_{\nu}=0$ then $x_{\nu}$ has a subsequence with limit 0.

There then exists $\varepsilon_{1}>0$, such that for all $0<\varepsilon<\varepsilon_{1}$ the operator $\Gamma_{\varepsilon}$ is injective and its inverse as an operator from its range $R_{\varepsilon}$ to $E$ satisfies $\left\|\Gamma_{\varepsilon}^{-1}\right\|_{\mathscr{L}\left(R_{\varepsilon}, E\right)} \leq K$ where the constant $K$ is independent of $\varepsilon$.

Proof of Lemma 3.2. Clearly there exists $\varepsilon_{1}$ such that $\Gamma_{\varepsilon}$ is injective for $0<$ $\varepsilon<\varepsilon_{1}$. If it did not exist there would exist a sequence $\varepsilon_{\nu} \rightarrow 0$ and a sequence of unit vectors $x_{\nu}$ such that $\Gamma_{\varepsilon_{\nu}} x_{\nu}=0$, contradicting $(\mathrm{C})$.

Suppose the bound on the inverse does not hold for any $\varepsilon_{1}$ and $K$. Then we can find a sequence $\varepsilon_{\nu} \rightarrow 0$ and a sequence of unit vectors $y_{\nu}=\Gamma_{\varepsilon_{\nu}} x_{\nu}$ in the range $R_{\varepsilon_{\nu}}$ such that $\left\|x_{\nu}\right\| \rightarrow \infty$. Let $v_{\nu}=x_{\nu} /\left\|x_{\nu}\right\|$. Then $\Gamma_{\varepsilon_{\nu}} v_{\nu}=y_{\nu} /\left\|x_{\nu}\right\| \rightarrow 0$, contradicting (C). This ends the proof of Lemma 3.2.

We continue with step 2 of the proof of Theorem 3.1. Let $\varepsilon_{\nu} \rightarrow 0$, let $v_{\nu}$ be a bounded sequence in $H^{2}$ and assume that

$$
\varepsilon_{\nu}^{-2}\left(-\Delta+\frac{\partial F}{\partial u}\left(V\left(\varepsilon_{\nu} x\right), u_{\varepsilon_{\nu}}\right)\right) v_{\nu} \rightarrow 0
$$


in $L^{2}$. We recall the asymptotic form for $u_{\varepsilon}$ (Theorem 2.6) and apply it to $u_{\varepsilon_{\nu}}$. For notational convenience, we abbreviate $s_{\varepsilon_{\nu}}$ to $s_{\nu}$ and $w_{\varepsilon_{\nu}}$ to $w_{\nu}$. We now replace $x$ by $x+\frac{b}{\varepsilon_{\nu}}-s_{\nu}$ and find

$$
\varepsilon_{\nu}^{-2}\left(-\Delta+\frac{\partial F}{\partial u}\left(V\left(\varepsilon_{\nu}\left(x-s_{\nu}\right)+b\right), \phi_{a}+\varepsilon_{\nu}^{2} w_{\nu}\right)\right) v_{\nu}\left(x+\frac{b}{\varepsilon_{\nu}}-s_{\nu}\right) \rightarrow 0
$$

in $L^{2}$.

We recall that our objective is to show that a subsequence of $v_{\nu}$ tends to 0 in $H^{2}$. In the sequel we shall repeatedly select a subsequence and always denote it by $v_{\nu}$ (and similarly for $\varepsilon_{\nu}$ etc.) using a phrase such as "going to a subsequence we may assume".

Let $W$ be the subspace of $H^{2}$ orthogonal in the $L^{2}$ sense to the $n$ partial derivatives $D_{j} \phi_{a}$. We write

$$
v_{\nu}\left(\cdot+\frac{b}{\varepsilon_{\nu}}-s_{\nu}\right)=\sigma_{\nu} \cdot \nabla \phi_{a}+\gamma_{\nu} h_{\nu}
$$

where $\sigma_{\nu} \in \mathbf{R}^{n}, h_{\nu} \in W,\left\|h_{\nu}\right\|_{H^{2}}=1$ and $\gamma_{\nu} \geq 0$. Step 2 of the proof will be completed by showing that, after going to a subsequence, $\sigma_{\nu}$ and $\gamma_{\nu}$ both have the limit 0 .

We begin by observing that since $v_{\nu}$ is bounded in $H^{2}$ the sequences $\gamma_{\nu}$ and $\sigma_{\nu}$ are bounded. We may therefore assume (going to a subsequence) that $\gamma_{\nu} \rightarrow \gamma_{0}, \sigma_{\nu} \rightarrow \sigma_{0}$ and, exploiting weak compactness of the unit ball in Hilbert space, that $h_{\nu} \rightarrow h_{0}$ weakly in $H^{2}$.

In view of the equation $\left(-\Delta+\frac{\partial F}{\partial u}\left(a, \phi_{a}\right)\right) \nabla \phi_{a}=0$ we now have

$$
\begin{aligned}
& \varepsilon_{\nu}^{-2} \gamma_{\nu}\left(-\Delta+\frac{\partial F}{\partial u}\left(V\left(\varepsilon\left(x-s_{\nu}\right)+b\right), \phi_{a}+\varepsilon_{\nu}^{2} w_{\nu}\right)\right) h_{\nu} \\
& +\varepsilon_{\nu}^{-2}\left(\frac{\partial F}{\partial u}\left(V\left(\varepsilon_{\nu}\left(x-s_{\nu}\right)+b\right), \phi_{a}+\varepsilon_{\nu}^{2} w_{\nu}\right)-\frac{\partial F}{\partial u}\left(a, \phi_{a}\right)\right)\left(\nabla \phi_{a} \cdot \sigma_{\nu}\right) \rightarrow 0
\end{aligned}
$$

in $L^{2}$. It is our objective to show that the left-hand side of (3.1) has a computable limit which gives rise to a limit equation. The computation will occupy the bulk of step 2 of the proof of Theorem 3.1 and concludes with the limit equation (3.6).

We first claim that the second term in (3.1) tends in $L^{2}$ to

$$
\frac{\partial^{2} F}{\partial u^{2}}\left(a, \phi_{a}\right) \eta \nabla \phi_{a} \cdot \sigma_{0}+\frac{1}{2} \frac{\partial^{2} F}{\partial u \partial a}\left(a, \phi_{a}\right)(H(b) x \cdot x) \nabla \phi_{a} \cdot \sigma_{0}
$$

where $H(b)$ is the Hessian matrix of $V$ and $\eta$ is the solution to (2.1) (see Theorem 2.6). To see this we expand it into

$$
\begin{aligned}
& \varepsilon_{\nu}^{-2}\left(\frac{\partial F}{\partial u}\left(V\left(\varepsilon_{\nu}\left(x-s_{\nu}\right)+b\right), \phi_{a}+\varepsilon_{\nu}^{2} w_{\nu}\right)-\frac{\partial F}{\partial u}\left(V\left(\varepsilon_{\nu}\left(x-s_{\nu}\right)+b\right), \phi_{a}\right)\right)\left(\nabla \phi_{a} \cdot \sigma_{\nu}\right) \\
& +\varepsilon_{\nu}^{-2}\left(\frac{\partial F}{\partial u}\left(V\left(\varepsilon_{\nu}\left(x-s_{\nu}\right)+b\right), \phi_{a}\right)-\frac{\partial F}{\partial u}\left(a, \phi_{a}\right)\right)\left(\nabla \phi_{a} \cdot \sigma_{\nu}\right)
\end{aligned}
$$

The first summand of (3.2) can be written as the integral

$$
\int_{0}^{1} \frac{\partial^{2} F}{\partial u^{2}}\left(V\left(\varepsilon_{\nu}\left(x-s_{\nu}\right)+b\right), \phi_{a}+\tau \varepsilon_{\nu}^{2} w_{\nu}\right) w_{\nu} \nabla \phi_{a} \cdot \sigma_{\nu} d \tau
$$


For fixed $\tau \in[0,1]$

$$
\frac{\partial^{2} F}{\partial u^{2}}\left(V\left(\varepsilon_{\nu}\left(x-s_{\nu}\right)+b\right), \phi_{a}+\tau \varepsilon_{\nu}^{2} w_{\nu}\right) \eta \nabla \phi_{a} \cdot \sigma_{0} \longrightarrow \frac{\partial^{2} F}{\partial u^{2}}\left(a, \phi_{a}\right) \eta \nabla \phi_{a} \cdot \sigma_{0}
$$

according to Lemma 2.2. Moreover,

$$
\begin{aligned}
& \frac{\partial^{2} F}{\partial u^{2}}\left(V\left(\varepsilon_{\nu}\left(x-s_{\nu}\right)+b\right), \phi_{a}+\tau \varepsilon_{\nu}^{2} w_{\nu}\right) w_{\nu}\left(\nabla \phi_{a} \cdot \sigma_{\nu}\right) \\
& -\frac{\partial^{2} F}{\partial u^{2}}\left(V\left(\varepsilon_{\nu}\left(x-s_{\nu}\right)+b\right), \phi_{a}+\tau \varepsilon_{\nu}^{2} w_{\nu}\right) \eta\left(\nabla \phi_{a} \cdot \sigma_{0}\right)
\end{aligned}
$$

tends to 0 owing to Lemma 2.1, the boundedness of $V$, and the limits $w_{\nu} \rightarrow \eta$ in $H^{2}$ and $\nabla \phi_{a} \cdot \sigma_{\nu} \rightarrow \nabla \phi_{a} \cdot \sigma_{0}$ in $H^{2}$. Therefore, the integrand in (3.3) tends to

$$
\frac{\partial^{2} F}{\partial u^{2}}\left(a, \phi_{a}\right) \eta \nabla \phi_{a} \cdot \sigma_{0}
$$

in $L^{2}$ at fixed $\tau$. Also, the $L^{2}$-norm of the integrand stays bounded independently of $\tau$ and $\nu$ (again by Lemma 2.1), so the dominated convergence theorem for $L^{2}$-valued integrals shows that the integral tends in $L^{2}$ to

$$
\frac{\partial^{2} F}{\partial u^{2}}\left(a, \phi_{a}\right) \eta \nabla \phi_{a} \cdot \sigma_{0}
$$

The second summand of (3.2) can be written as the integral

$$
\varepsilon_{\nu}^{-1} \int_{0}^{1} \frac{\partial^{2} F}{\partial u \partial a}\left(V\left(\tau \varepsilon_{\nu}\left(x-s_{\nu}\right)+b, \phi_{a}\right)\left(\nabla \phi_{a} \cdot \sigma_{\nu}\right) \nabla V\left(\tau \varepsilon_{\nu}\left(x-s_{\nu}\right)+b\right) \cdot\left(x-s_{\nu}\right) d \tau\right.
$$

Since, by assumption, $\nabla V(b)=0$, this is equal to

$$
\int_{0}^{1} \int_{0}^{1} \frac{\partial^{2} F}{\partial u \partial a}\left(V\left(\tau \varepsilon_{\nu}\left(x-s_{\nu}\right)+b, \phi_{a}\right)\left(\nabla \phi_{a} \cdot \sigma_{\nu}\right) H\left(\rho \tau \varepsilon_{\nu}\left(x-s_{\nu}\right)+b\right)\left(x-s_{\nu}\right) \cdot\left(x-s_{\nu}\right) \tau d \rho d \tau\right.
$$

Now, $H(x)$ has polynomial growth, $\frac{\partial^{2} F}{\partial u \partial a}\left(V\left(\tau \varepsilon_{\nu}\left(x-s_{\nu}\right)+b, \phi_{a}\right)\right.$ is bounded uniformly with respect to $\tau$ and $\nu$ as $x$ goes to infinity, because of our growth conditions, and $\left(\nabla \phi_{a} \cdot \sigma_{\nu}\right)$ has uniform exponential decay as $x$ goes to infinity (since $\sigma_{\nu}$ is a bounded sequence). Hence, for fixed $\tau$, the integrand converges to

$$
\frac{\partial^{2} F}{\partial u \partial a}\left(a, \phi_{a}\right)\left(\nabla \phi_{a} \cdot \sigma_{0}\right)(H(b) x \cdot x) \tau
$$

in $L^{2}$, and is also bounded uniformly with respect to $\tau$ and $\nu$ by a fixed function in $L^{2}$. We can therefore apply the dominated convergence theorem for $L^{2}$-valued integrals and the double integral tends to

$$
\frac{1}{2} \frac{\partial^{2} F}{\partial u \partial a}\left(a, \phi_{a}\right)\left(\nabla \phi_{a} \cdot \sigma_{0}\right)(H(b) x \cdot x) .
$$

This proves our claim that the second term of (3.1) has a limit in $L^{2}$.

Next we consider the first term of (3.1). We claim that the multiplier $\varepsilon_{\nu}^{-2} \gamma_{\nu}$ is bounded. Suppose, for the sake of contradiction, that $\varepsilon_{\nu}^{-2} \gamma_{\nu}$ is unbounded. Going to a subsequence we may assume that $\varepsilon_{\nu}^{2} \gamma_{\nu}^{-1} \rightarrow 0$ and multiplying (3.1) by it and knowing that the second term of (3.1) converges in $L^{2}$, we obtain

$$
\left(-\Delta+\frac{\partial F}{\partial u}\left(V\left(\varepsilon_{\nu}\left(x-s_{\nu}\right)+b\right), \phi_{a}+\varepsilon_{\nu}^{2} w_{\nu}\right)\right) h_{\nu} \rightarrow 0
$$


in $L^{2}$. Now we have

$$
\frac{\partial F}{\partial u}\left(a, \phi_{a}\right) h_{\nu} \rightarrow \frac{\partial F}{\partial u}\left(a, \phi_{a}\right) h_{0}
$$

weakly in $L^{2}$ since multiplication by $\frac{\partial F}{\partial u}\left(a, \phi_{a}\right)$ is a norm-continuous linear operator from $H^{2}$ to $L^{2}$. Moreover

$$
\left(\frac{\partial F}{\partial u}\left(V\left(\varepsilon_{\nu}\left(x-s_{\nu}\right)+b\right), \phi_{a}+\varepsilon_{\nu}^{2} w_{\nu}\right)-\frac{\partial F}{\partial u}\left(a, \phi_{a}\right)\right) h_{\nu} \rightarrow 0
$$

in the weak topology of the dual of $H^{2}$ by Lemma 2.3 . Finally $\Delta h_{\nu} \rightarrow \Delta h_{0}$ in the sense of distributions. We conclude from (3.4) that

$$
-\Delta h_{0}+\frac{\partial F}{\partial u}\left(a, \phi_{a}\right) h_{0}=0 .
$$

But $h_{0} \in W$ so that $h_{0}=0$ by property 4 of $\phi_{a}$. We deduce by Lemma 2.4 that

$$
\left(\frac{\partial F}{\partial u}\left(V\left(\varepsilon_{\nu}\left(x-s_{\nu}\right)+b\right), \phi_{a}+\varepsilon_{\nu}^{2} w_{\nu}\right)-\frac{\partial F}{\partial u}\left(V\left(\varepsilon_{\nu}\left(x-s_{\nu}\right)+b\right), 0\right)\right) h_{\nu} \rightarrow 0
$$

in $L^{2}$, so that now (3.4) yields

$$
\left(-\Delta+\frac{\partial F}{\partial u}\left(V\left(\varepsilon_{\nu}\left(x-s_{\nu}\right)+b\right), 0\right)\right) h_{\nu} \rightarrow 0
$$

in $L^{2}$. Now the positivity assumption and Wang's Lemma give $h_{\nu} \rightarrow 0$ in $H^{2}$ thus contradicting the assumption that $\left\|h_{\nu}\right\|_{H^{2}}=1$. This contradiction implies that $\varepsilon_{\nu}^{-2} \gamma_{\nu}$ is bounded as claimed. One consequence of this is that $\gamma_{\nu} \rightarrow 0$; thus one of the objectives of step 2 is attained.

We may now assume, going once more to a subsequence, that $\varepsilon_{\nu}^{-2} \gamma_{\nu} \rightarrow c \geq 0$. Armed with this knowledge we return to (3.1) reminding ourselves at this point that now we do not necessarily have $h_{0}=0$. We do, however, still have that

$$
\left(-\Delta+\frac{\partial F}{\partial u}\left(V\left(\varepsilon_{\nu}\left(x-s_{\nu}\right)+b\right), \phi_{a}+\varepsilon_{\nu}^{2} w_{\nu}\right)\right) h_{\nu} \rightarrow\left(-\Delta+\frac{\partial F}{\partial u}\left(a, \phi_{a}\right)\right) h_{0}
$$

in the sense of distributions. Passing to the limit in (3.1) we find that

$$
\begin{aligned}
& \left(-\Delta+\frac{\partial F}{\partial u}\left(a, \phi_{a}\right)\right) c h_{0}+\frac{\partial^{2} F}{\partial u^{2}}\left(a, \phi_{a}\right) \eta\left(\nabla \phi_{a} \cdot \sigma_{0}\right) \\
& +\frac{1}{2} \frac{\partial^{2} F}{\partial u \partial a}\left(a, \phi_{a}\right)(H(b) x \cdot x) \nabla \phi_{a} \cdot \sigma_{0}=0 .
\end{aligned}
$$

Now recall that $\eta$ satisfies the equation

$$
-\Delta \eta+\frac{\partial F}{\partial u}\left(a, \phi_{a}\right) \eta=-\frac{1}{2} \frac{\partial F}{\partial a}\left(a, \phi_{a}\right)(H(b) x \cdot x) .
$$

Differentiating this with respect to $x$ gives the vector-valued equation

$$
\begin{aligned}
& -\Delta(\nabla \eta)+\frac{\partial F}{\partial u}\left(a, \phi_{a}\right) \nabla \eta+\frac{\partial^{2} F}{\partial u^{2}}\left(a, \phi_{a}\right) \eta \nabla \phi_{a} \\
& =-\frac{1}{2} \frac{\partial^{2} F}{\partial a \partial u}\left(a, \phi_{a}\right)(H(b) x \cdot x) \nabla \phi_{a}-\frac{\partial F}{\partial a}\left(a, \phi_{a}\right) H(b) x .
\end{aligned}
$$


Taking the inner product with $\sigma_{0}$ and using (3.5) gives the final form of the limit equation that arises from (3.1):

$$
\left(-\Delta+\frac{\partial F}{\partial u}\left(a, \phi_{a}\right)\right)\left(c h_{0}+\nabla \eta \cdot \sigma_{0}\right)=-\frac{\partial F}{\partial a}\left(a, \phi_{a}\right) H(b) x \cdot \sigma_{0} .
$$

The next objective is to show that $\sigma_{0}=0$. By (3.6) the function $\frac{\partial F}{\partial a}\left(a, \phi_{a}\right) H(b) x \cdot \sigma_{0}$ is in the range of $-\Delta+\frac{\partial F}{\partial u}\left(a, \phi_{a}\right)$, which is the space $W$. It follows that $\sigma_{0}$ satisfies the linear equation system

$$
\int\left(\frac{\partial F}{\partial a}\left(a, \phi_{a}\right) H(b) x \cdot \sigma_{0}\right) D_{j} \phi_{a}(x) d x=0, \quad j=1, \ldots, n .
$$

We claim that this implies $\sigma_{0}=0$. Write $\sigma_{0}=\left(\sigma_{1}, \ldots, \sigma_{n}\right)$. The inner product $H(b) x \cdot \sigma_{0}$ is given by

$$
H(b) x \cdot \sigma_{0}=\sum_{i, k} \sigma_{i}\left(x_{k} H_{k, i}\right)
$$

where the $H_{k, i}$ are the coefficients of the matrix $H(b)$. Moreover, $\phi_{a}$ is spherically symmetric, $\phi_{a}(x)=\Phi_{a}(r)$, so our system can be written as

$$
\int\left(\sum_{i, k} \sigma_{i}\left(x_{k} H_{k, i}\right)\right) \frac{\partial F}{\partial a}\left(a, \Phi_{a}(r)\right) \frac{\Phi_{a}^{\prime}(r) x_{j}}{r} d x=0, \quad j=1, \ldots, n .
$$

Spherical symmetry causes terms involving mixed products $x_{j} x_{k}, j \neq k$, to vanish, leading to the simpler equation

$$
\left(\sum_{i} \sigma_{i} H_{j, i}\right) \int \frac{\partial F}{\partial a}\left(a, \Phi_{a}(r)\right) \Phi_{a}^{\prime}(r) \frac{x_{j}^{2}}{r} d x=0
$$

for all $j$. It is easily seen that the integral is independent of $j$, and so its value is

$$
C:=\frac{1}{n} \int \frac{\partial F}{\partial a}\left(a, \Phi_{a}(r)\right) \Phi_{a}^{\prime}(r) r d x
$$

which is non-zero by assumption. Therefore, our system reduces to

$$
C \sum_{i} \sigma_{i} H_{j, i}=0
$$

for all $j$, and since $H(b)$ is an invertible matrix, this implies $\sigma_{0}=0$, hence our claim.

We have now arrived at a subsequence $v_{\nu}$ for which $\sigma_{\nu} \rightarrow 0$ and $\varepsilon_{\nu}^{-2} \gamma_{\nu}$ is bounded. The latter implies $\gamma_{\nu} \rightarrow 0$. Since $v_{\nu}\left(\cdot+\frac{b}{\varepsilon_{\nu}}-s_{\nu}\right)=\nabla \phi_{a} \cdot \sigma_{\nu}+\gamma_{\nu} h_{\nu}$ and $\left\|h_{\nu}\right\|_{H^{2}}=1$ we conclude that $v_{\nu} \rightarrow 0$ in $H^{2}$. This concludes step 2 of the proof and therefore the whole proof is complete.

We remark that for $c<2$ it is not the case that $\left\|T_{\varepsilon}^{-1}\right\|=O\left(\varepsilon^{-c}\right)$. Indeed if $c<2$ then $\lim _{\varepsilon \rightarrow 0} \varepsilon^{-c} T_{\varepsilon} v_{\varepsilon}=0$ in $L^{2}$, where $v_{\varepsilon}(x)=D_{j} \phi_{a}\left(x-\frac{b}{\varepsilon}+s_{\varepsilon}\right)$.

\section{Remark on the assumptions}

The non-vanishing of the integral

$$
I:=\int \frac{\partial F}{\partial a}\left(a, \Phi_{a}(r)\right) \Phi_{a}^{\prime}(r) r d x
$$


was used in the proof of Theorem 3.1 (as well as in the proof of the very existence of $u_{\varepsilon}$ ). This condition may seem rather technical and meaningless. We can however derive it from a rather natural assumption in which the continuum of ground states $\phi_{a}$ plays an interesting role. We shall assume that the $\phi_{a}$ form a smooth continuum with respect to $a$, an assumption that we did not need before, since we were always working at fixed $a$. This is often the case since, in practice, the functions $\phi_{a}$ are usually obtained by scaling from the case $a=1$.

Proposition 4.1. In addition to all previous hypotheses on $\phi_{a}$, assume that it is a $C^{1}$ function of $a$. Then the integral $I$ is non-zero if and only if

$$
\frac{d}{d a}\left(\int\left|\nabla \phi_{a}\right|^{2} d x\right) \neq 0
$$

Proof. We shall in fact establish the identity

$$
I:=\int \frac{\partial F}{\partial a}\left(a, \Phi_{a}(r)\right) \Phi_{a}^{\prime}(r) r d x=-\frac{d}{d a}\left(\int\left|\nabla \phi_{a}\right|^{2} d x\right) .
$$

By spherical symmetry we have

$$
\int \frac{\partial F}{\partial a}\left(a, \Phi_{a}(r)\right) \Phi_{a}^{\prime}(r) r d x=n \int \frac{\partial F}{\partial a}\left(a, \phi_{a}(x)\right) x_{j} D_{j} \phi_{a}(x) d x
$$

for each $j$. Differentiating the equation $-\Delta \phi_{a}+F\left(a, \phi_{a}\right)=0$ with respect to $a$ gives

$$
-\Delta\left(\frac{\partial \phi_{a}}{\partial a}\right)+\frac{\partial F}{\partial u}\left(a, \phi_{a}\right) \frac{\partial \phi_{a}}{\partial a}+\frac{\partial F}{\partial a}\left(a, \phi_{a}\right)=0
$$

and therefore

$$
\begin{aligned}
I & =n \int\left(\Delta-\frac{\partial F}{\partial u}\left(a, \phi_{a}\right)\right)\left(\frac{\partial \phi_{a}}{\partial a}\right) x_{j} D_{j} \phi_{a}(x) d x \\
& =n \int \frac{\partial \phi_{a}}{\partial a}\left(\Delta-\frac{\partial F}{\partial u}\left(a, \phi_{a}\right)\right)\left(x_{j} D_{j} \phi_{a}\right) d x
\end{aligned}
$$

using self-adjointness. We expand

$$
\Delta\left(x_{j} D_{j} \phi_{a}\right)=x_{j} \Delta D_{j} \phi_{a}+2 D_{j}^{2} \phi_{a}
$$

and since the partial derivatives $D_{j} \phi_{a}$ belong to the kernel of $-\Delta+\frac{\partial F}{\partial u}\left(a, \phi_{a}\right)$, we are left with

This is true for each $j$, so

$$
I=2 n \int \frac{\partial \phi_{a}}{\partial a} D_{j}^{2} \phi_{a} d x
$$

$$
I=2 \int \frac{\partial \phi_{a}}{\partial a} \Delta \phi_{a} d x=-2 \int \nabla \phi_{a} \cdot \nabla\left(\frac{\partial \phi_{a}}{\partial a}\right) d x=-\frac{d}{d a}\left(\int\left|\nabla \phi_{a}\right|^{2} d x\right),
$$

and the proof is complete.

We now give more details about how a continuum can be obtained from a single solution for a fixed value of $a$, and how the result of the previous proposition applies then.

Assume we are given a non-trivial, spherically symmetric solution $\psi(x)$ in $H^{2}$ of an equation

$$
-\Delta u+G(u)=0
$$


where $G$ satisfies our regularity and growth conditions. For $a$ in a bounded interval $I$, we put

$$
\phi_{a}(x)=a^{\mu} \psi\left(a^{\nu} x\right)
$$

for positive exponents $\mu$ and $\nu$. This is obviously smooth with respect to $a$. Moreover,

$$
\Delta \phi_{a}=a^{\mu+2 \nu} \Delta \psi\left(a^{\nu} x\right)=a^{\mu+2 \nu} G\left(a^{-\mu} \phi_{a}\right) .
$$

Therefore $\phi_{a}$ solves $-\Delta u+F(a, u)=0$ where

$$
F(a, u)=a^{\mu+2 \nu} G\left(a^{-\mu} u\right) .
$$

We see that $F(1, u)=G(u)$. The function $F$ satisfies our growth conditions. According to Proposition 4.1, the non-vanishing of the integral 4.1 reduces to

$$
0 \neq \frac{d}{d a}\left(a^{2 \mu+2 \nu} \int\left|\nabla \psi\left(a^{\nu} x\right)\right|^{2} d x\right)=(2 \mu+2 \nu-n \nu) a^{2 \mu+2 \nu-n \nu-1} \int|\nabla \psi|^{2} d x .
$$

So a necessary and sufficient condition for the non-vanishing of this integral is simply

$$
2 \mu+2 \nu-n \nu \neq 0 \text {. }
$$

Under this simple assumption, we can apply Theorems 2.6 and 3.1, using a potential $V$ with range in $I$.

\section{References}

[1] Angenent, S.: The shadowing lemma for elliptic PDE. - In: Dynamics of infinite-dimensional systems, edited by S. N. Chow and J. K. Hale, Springer-Verlag, New York, 1987, 7-22.

[2] Kwong, M. K.: Uniqueness of positive solutions of $\Delta u-u+u^{p}=0$ in $\mathbf{R}^{n}$. - Arch. Ration. Mech. Anal. 105, 1989, 243-266.

[3] Magnus, R.: The implicit function theorem and multibump solutions of periodic partial differential equations. - Proc. Roy. Soc. Edinburgh Sect. A 136, 2006, 559-583.

[4] Magnus, R.: A scaling approach to bumps and multi-bumps for nonlinear partial differential equations. - Proc. Roy. Soc. Edinburgh Sect. A 136, 2006, 585-614.

[5] Strauss, W. A.: Existence of solitary waves in higher dimensions. - Comm. Math. Phys. 55:2, 1977, 149-162.

[6] WANG, X.: On concentration of positive bound states of nonlinear Schrödinger equations. Comm. Math. Phys. 153, 1993, 229-244.

[7] Weinstein, M.: Modulational stability of the ground states of nonlinear Schrödinger equations. - SIAM J. Math. Anal. 16, 1985, 567-576.

Received 11 July 2008 